\title{
Low birth weight is associated with impaired murine kidney development and function
}

Christina Barnett ${ }^{1}$, Oluwadara Nnoli ${ }^{2}$, Wasan Abdulmahdi ${ }^{2}$, Lauren Nesi ${ }^{1}$, Michael Shen ${ }^{1}$, Joseph A. Zullo ${ }^{2}$, David L. Payne ${ }^{1}$, Tala Azar ${ }^{1}$, Parth Dwivedi ${ }^{2}$, Kunzah Syed ${ }^{1}$, Jonathan Gromis ${ }^{1}$, Mark Lipphardt ${ }^{1}$, Edson Jules ${ }^{1}$, Eric L. Maranda ${ }^{3}$, Amy Patel $^{1}$, May M. Rabadi ${ }^{4}$ and Brian B. Ratliff ${ }^{1}$

BACKGROUND: Low birth weight (LBW) neonates have impaired kidney development that leaves them susceptible to kidney disease and hypertension during adulthood. The study here identifies events that blunt nephrogenesis and kidney development in the murine LBW neonate.

METHODS: We examined survival, kidney development, GFR, gene expression, and cyto-/chemokines in the LBW offspring of malnourished (caloric and protein-restricted) pregnant mice.

RESULTS: Malnourished pregnant mothers gave birth to LBW neonates that had 40\% reduced body weight and 54\% decreased survival. Renal blood perfusion was reduced by $37 \%$, whereas kidney volume and GFR were diminished in the LBW neonate. During gestation, the LBW neonatal kidney had 2.2-fold increased apoptosis, 76\% decreased SIX2+ progenitor cells, downregulation of mesenchymal-to-epithelial signaling factors Wnt9b and Fgf8, 64\% less renal vesicle formation, and $32 \%$ fewer nephrons than controls. At birth, increased plasma levels of $I L-1 \beta, I L-6, I L-12(p 70)$, and granulocyte-macrophage colony-stimulating factor in the LBW neonate reduced SIX2+ progenitor cells.

CONCLUSION: Increased pro-inflammatory cytokines in the LBW neonate decrease SIX2+ stem cells in the developing kidney. Reduced renal stem cells (along with the decreased mesenchymal-to-epithelial signaling) blunt renal vesicle generation, nephron formation, and kidney development. Subsequently, the mouse LBW neonate has reduced glomeruli volume, renal perfusion, and GFR.

$I^{\mathrm{n}}$ n the United States, $~ 8 \%$ of all live human births are of low birth weight (LBW) infants $(<2,500 \mathrm{~g})(1)$. LBW infants are a mixed group representing full-term babies with growth retardation/subnormal weight and infants born premature ( $<37$ weeks gestation). A significant cause of low infant birth weight is maternal malnourishment during gestation, which results in underdeveloped kidney and vascular systems, and a reduced final nephron complement. In addition to acute problems soon after birth, these babies often have problems during their adult years with high risk for heart disease, hypertension, stroke, impaired immunity, diabetes, and chronic kidney disease (2-6). In regard to chronic kidney disease, studies show that LBW in humans is a risk factor for the development of focal segmental glomerular sclerosis and is associated with a $70 \%$ increase in the risk of end-stage kidney failure in adults $(4,7)$.

Despite the recent recognition of the short- and long-term risks that LBW poses, our understanding of the underlying causes of impaired renal developmental in these neonates is extremely limited. Thus, in the study here, we investigated the underlying causes that are associated with impaired renal development in the maternal malnourished-induced murine LBW neonate. To generate LBW mice neonates, pregnant mice were maintained on a malnourished diet during the second half of gestation, which consisted of a combined low-protein diet (6\% protein) and a $50 \%$ reduction in their total daily caloric intake. This diet resulted in pregnant dams that gave birth to LBW male and female offspring with impaired kidney development. We first examined survival, renal blood perfusion, and GFR in these LBW neonates. As it is unknown as to which stages of nephrogenesis are impaired in the developing LBW neonatal kidney leading to reduced nephron generation, we next identified and characterized the critical time course of events that leads to blunted nephrogenesis in the LBW neonate. Our investigation identified decreased formation of early nephron progenitor structures (renal vesicles) in the LBW neonatal kidney, and when the insufficiency in nephron formation occurs during kidney development.

How endogenous stem cell pools are affected in the developing kidney of the LBW neonate remains ambiguous. Hence, we sought to determine whether the underlying cause of nephrogenesis impairment in the LBW neonate is due to a decrease in SIX2+ stem cells in the metanephric mesenchyme of the developing kidney. SIX2+ stem cells are critical progenitor cells that are required for the generation of renal vesicles and formation of nephrons during kidney development. A reduction in the quantity and/or competence of these cells for any reason

\footnotetext{
${ }^{1}$ Department of Medicine, Renal Research Institute, New York Medical College, Valhalla, New York; ${ }^{2}$ Department of Physiology, Renal Research Institute, New York Medical College, Valhalla, New York; ${ }^{3}$ Department of Dermatology and Cutaneous Surgery, Miami University, Miami, Florida; ${ }^{4}$ Department of Anesthesiology, College of Physicians and Surgeons of Columbia University, New York, New York. Correspondence: Brian B. Ratliff (Ratlifbb@gmail.com) 
reduces nephrogenesis (8). We hypothesized that, in the LBW neonate, there is a reduction in the quantity and quality of SIX2 + stem cells in the metanephric mesenchyme, which consequently reduces the formation of renal vesicles and nephrons. We also rationalized that there is potential upregulation of proinflammatory cyto-/chemokines in the developing LBW neonate because of the metabolic stress suffered by the malnourished pregnant mother, and the stress placed on the LBW neonate's underdeveloped vascular and renal systems to function prior to their adequate development. In turn, we hypothesized that such potential upregulation of cyto-/chemokines compromises SIX2+ stem cell populations in the developing kidney and subsequently blunts kidney development. These phenomena were examined in the study here.

\section{METHODS}

\section{Animals}

The animal study protocol was in accordance with the NIH's Guide for the Care and Use of Laboratory Animals and was approved by the Institutional Animal Care and Use Committee. Pregnant CD-1 mice were obtained from Charles River Lab and used in all experiments. Animals were separately caged with a 12:12 h light-dark cycle and free access to water throughout the study. Gestation in a mouse typically lasts 19 days, with nephrogenesis occurring between embryonic/ gestation day 11 (E11) and 7 days post birth (P7). Pregnant mice were given free access to standard rodent chow (LabDiet, St Louis, MO) composed of $26 \%$ protein for the first 8 days of gestation. At day 9 of gestation, pregnant mice were started on the combined caloric and protein-restricted diet to induce malnourishment (control mice were maintained on the $26 \%$ protein chow throughout gestation with free access to the chow). To implement the food-restricted diet, mice were given only $50 \%$ (by weight) of the chow they would normally eat per day. In addition, the chow they were given had a low protein content ( $6 \%$ protein content; carbohydrates replaced the removed protein content) (Envigo, Indianapolis, IN). Pregnant mothers were maintained on the food-restricted diet throughout the rest of gestation. Food-restricted mothers consistently gave birth to LBW neonates on gestation day 19, with only slight fluctuations when, exactly on day 19, pup delivery occurred. No premature births from food-restricted mothers occurred during the study.

After birth, control and LBW neonates were sacrificed and sampled on postnatal days 0,3 , and 7 (P0, P3, and P7, respectively). For sampling, pups were anesthetized with $5 \%$ isoflurane inhalation and sacrificed via decapitation. After sacrifice, blood was collected from the carotid arteries, and the kidneys were excised. Samples were also obtained from embryos at E15 and E17. To obtain embryo samples, pregnant mothers were anesthetized (5\% isoflurane inhalation) followed by sacrifice via cervical dislocation. After sacrifice, a midline incision exposed the abdominal section, and uterine horns containing embryos were removed. Embryos were removed from the uterine horns and placentas, and they were decapitated to ensure sacrifice. After sacrifice, the kidneys were excised.

\section{Glomerular Volume}

After excision from animals, the kidneys were measured using a dissecting microscope. Kidney measurements included kidney length, anterior-posterior diameter, and transverse diameter. Using the obtained measurements, kidney volume was calculated as previously reported (9) using the equation for an ellipsoid (kidney volume = length $\times$ transverse diameter $\times$ anterior-posterior diameter).

\section{Renal Vesicle and Glomeruli Count}

To determine total nephron and renal vesicle number in kidneys, we used the physical dissector/fractionator stereological method previously described by Cullen-McEwen et al. $(10,11)$. In brief, kidneys were excised from animals, fixed in 4\% paraformaldehyde (PFA) (Electron Microscopy Sciences, Hatfield, PA), and embedded in paraffin. Using a microtome, $4 \mu \mathrm{m}$ serial kidney sections were cut. Sections were stained with biotinylated Arachis hypogea lectin peanut agglutinin (PNA) (Sigma-Aldrich, St Louis, MO), followed by incubation with avidin/biotin complex and developed with DAB. Sections were counterstained with hematoxylin (Sigma-Aldrich). Stained kidney sections were imaged on a Nikon Eclipse TE 2000-U microscope (Morrell, Melville, NY) equipped with a 1.40 and a SPOT Insight QE color camera (model 4.2). The acquisition software used was SPOT imaging softwarem (version 4.0.1). Images of the cortex were captured in sequence at $\times 100$. Glomeruli and renal vesicles were identified and counted in sections using the physical dissector principle, in which identical regions in section pairs were compared and examined. Total glomeruli and renal vesicles were then calculated using the physical dissector/fractionator combination equation described by Cullen-McEwen et al. $(10,11)$.

\section{Immunofluorescence Analysis}

Kidneys were fixed in $4 \%$ PFA, dehydrated with $30 \%$ sucrose, and embedded in OCT (Sakura FineTek USA, Torrance, CA). After cryosectioning, $10 \mu \mathrm{m}$ thick sections were permeabilized with Triton $\mathrm{X}-100$ in $0.2 \% \mathrm{PBS} / \mathrm{BSA}$ (Sigma-Aldrich) and blocked with $0.2 \%$ PBS/BSA. Sections were incubated with rabbit anti-mouse primary antibody against SIX2 (dilution 1:350) (Proteintech, Chicago, IL) and goat anti-mouse primary antibody against calbindin (dilution 1:350) (Santa Cruz Biotechnology, Dallas, TX) overnight at $4{ }^{\circ} \mathrm{C}$. Alexa Fluor 594 donkey anti-rabbit and Alexa Fluor 488 donkey anti-goat secondary antibody (dilution 1:350) (Thermo Scientific, Grand Island, NY) were applied the following day for $1 \mathrm{~h}$. Following PBS washes, nuclei of cells were stained with $(10 \mathrm{mg} / \mathrm{ml}) 4^{\prime}$, 6-diamidino2-2phenylindole dihydrochloride (DAPI) (Sigma-Aldrich). Sections were examined for immunofluorescence using the previously described microscope equipment and software at $\times 400$ magnification. The total integrated density of SIX2 staining in images was quantified by NIH's ImageJ software and subsequently converted to cell number. This was done by dividing the total integrated density value by the integrated density obtained from a single SIX2+ cell. Fluorescence thresholds were set in ImageJ to avoid including background as false positives in the cell count. The cell count conversion was normalized by cortical area assessed in each respective image, as determined by ImageJ area analysis. To confirm the accuracy of cell counting by measurement of integrated density, positive SIX2 cells in images were also manually counted.

\section{TUNEL Staining}

Kidneys were fixed in 4\% PFA, dehydrated with $30 \%$ sucrose, and OCT-embedded. After cryosectioning, sections were examined for apoptosis using the In Situ Cell Death Detection (TUNEL) TMR red assay kit (Roche, Indianapolis, IN). In brief, OCT kidney cryosections $(10 \mu \mathrm{m}$ thick) were permeabilized with $0.2 \%$ Triton PBS/BSA, then incubated with the kit's TUNEL reaction mixture for $60 \mathrm{~min}$ at $37^{\circ} \mathrm{C}$. Total nuclei were stained with $(10 \mathrm{mg} / \mathrm{ml})$ DAPI. Positive controls were obtained by incubating sections with DNase I $(1: 100)$ (Qiagen, Valenica, CA) for $10 \mathrm{~min}$ at $15-25^{\circ} \mathrm{C}$ prior to the labeling procedures. Negative controls were obtained by incubating sections with the kit's labeling solution without terminal deoxynucleotidyl transferase. Staining was subsequently examined by immunofluorescence microscopy at $\times 400$ magnification. Cells that were positive for TUNEL staining in images were counted. TUNEL-positive cells were normalized to the total number of DAPI-positive cells in each image. To determine the total number of DAPI cells, the total area covered by positive DAPI staining was measured through ImageJ. To convert to total cell number, the total area of positive DAPI staining was divided by the area typically covered by a single DAPI-stained cell. To confirm whether the conversion of positive DAPI staining area to total cell number was accurate, we also manually counted DAPI-positive cells in images and compared the results. 


\section{Articles | Barnett et al.}

\section{Real-Time PCR: Gdnf, Wnt9b, and Fgf8 Expression}

mRNA from excised kidneys was extracted through the use of a commercially available kit (Denville Scientific, Inc., Holliston, MA). Kidneys were first homogenized via sonication (Misonix, Inc., Farmingdale, NY) in ice-cold lysis buffer provided by the kit. After homogenization, the kit's manufacturer's protocol was followed. At the conclusion of mRNA isolation, a reverse transcriptase kit (Applied Biosystems, Foster City, CA) was utilized to obtain cDNA sequences from isolated mRNA. cDNA was subsequently analyzed by real-time PCR using a commercially available SYBR green real-time PCR kit (Quanta Biosciences, Gaithersburg, MD) and the Stratagene Mx3000p real-time PCR instrument (Agilent Technologies, Santa Clara, CA) equipped with associated software $\mathrm{mx}$ pro qPCR v4.10. PCR primers (Thermo Scientific) for $G d n f$, $W n t 9 b, F g f 8$, and $18 s$ rRNA were utilized for quantification. Gdnf, $W n t 9 b$, and Fgf8 expression was normalized to $18 s$ rRNA expression. Primer sequences are listed in the Supplementary Data online.

\section{GFR Measurement}

GFR was measured in pups after birth using the NIC-Kidney GFR monitoring device (Mannheim Pharma \& Diagnostics, Mannheim, Germany) following the manufacturer's protocol and the protocols a

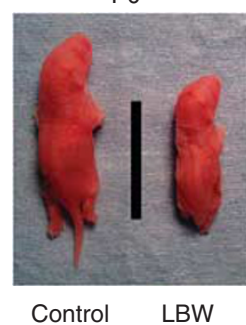

b

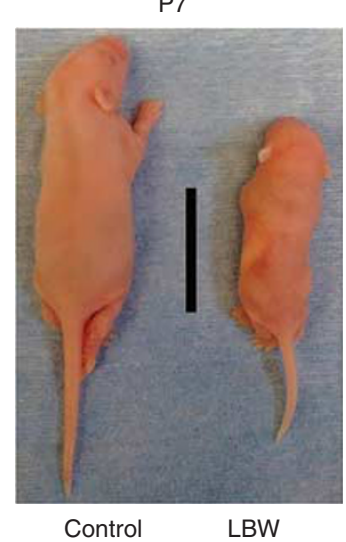

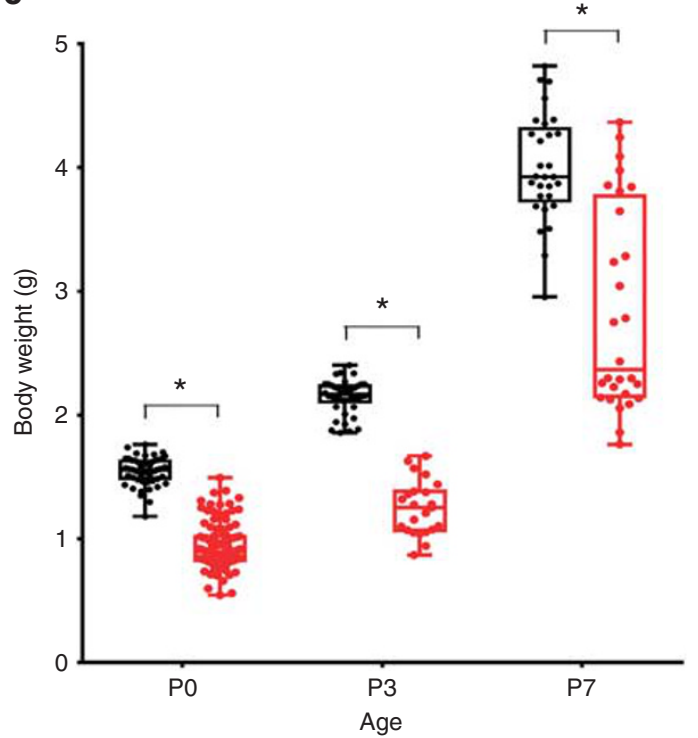

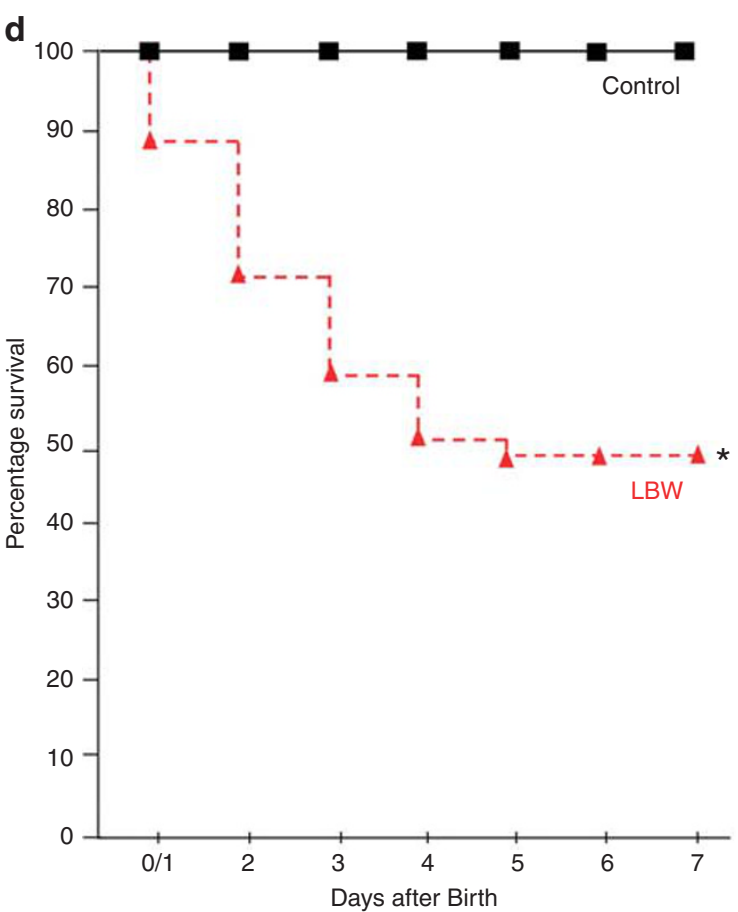

Figure 1. Body size, weight and survival of LBW neonates the first week after birth. (a) Image compares body size of control and LBW neonates on the day of birth (P0). (b) Image compares body size of control and LBW neonates 1 week after birth (P7). Bars $=2 \mathrm{~cm}$. (c) A comparison of control (black boxes and dots) and LBW neonate (red boxes and dots) body weights after birth. (d) The Kaplan-Meier survival curve compares the first week survival of control (black solid line) to LBW (red cross-hatched line) neonates. 


\section{The low birth weight kidney $\quad$ Articles}
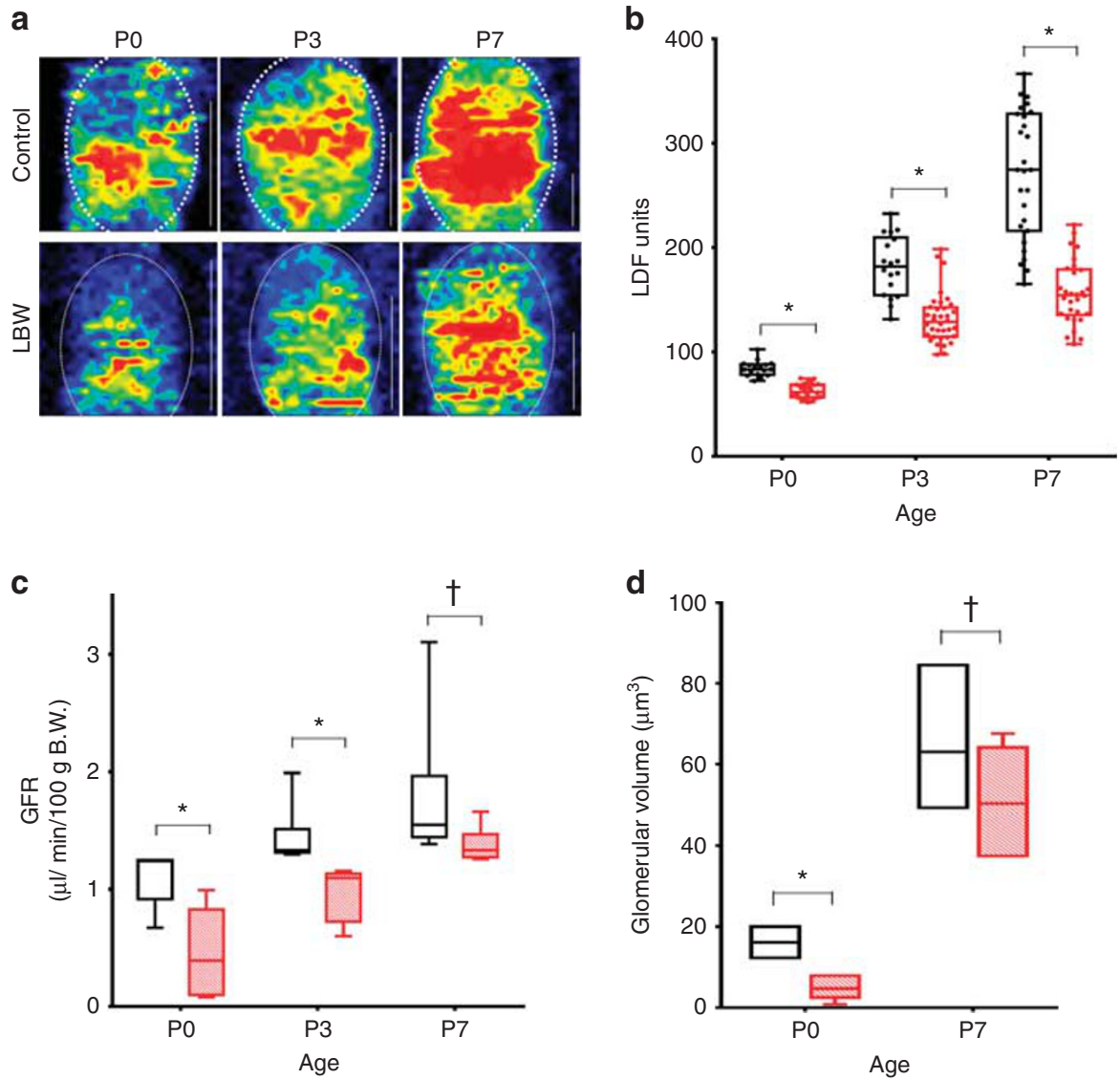

Figure 2. Renal blood perfusion, GFR and glomerular volume in LBW neonates the first week after birth. (a) Laser Doppler scans illustrate renal blood perfusion in control and LBW neonates. Increased flow is indicated by hotter colors (yellow to red), and decreased flow is indicated by cooler colors (green to blue). Bars $=100 \mu \mathrm{m}$. (b) Quantification of renal blood flow in control (black boxes and scatterplots) and LBW (red boxes and scatterplots) neonates after birth. (c) GFR in control (black boxes) and LBW (red boxes) neonates after birth. (d) Glomerular volume in control (black boxes) and LBW (red boxes) neonates after birth. ${ }^{\dagger} P \leqslant 0.10$, LBW vs. control.

previously described $(12,13)$. In short, pups were briefly sedated by inhalation of $5 \%$ isoflurane, followed by a single bolus injection of FITC-sinistrin (7 mg/100 g B.W.) (Fresenius Kabi, Linz, Austria). The NIC-kidney GFR monitoring device was positioned on the skin surface of the upper posterior thoracic area of pups for up to $60 \mathrm{~min}$ for transcutaneous measurement of FITC-sinistrin clearance.

\section{Cyto-/Chemokine Analysis}

Plasma obtained from neonates was analyzed for cyto-/chemokine content utilizing the Luminex 100 system (Luminex Corporation, Austin, TX) and an anti-mouse cyto-/chemokine panel I assay kit (Millipore, Billerica, MA), according to the manufacturer's protocol.

\section{Laser Doppler Flowmetry}

At varying time points after birth (P0, P3, and P7) and before sacrifice of animals, Laser Doppler flowmetry (Perimed, Jarfalla, Sweden) was utilized to determine kidney blood perfusion. Mice were anesthetized by $5 \%$ isoflurane inhalation. Before exposing the kidneys via laparotomy, a Laser Doppler flowmetry scanner obtained pseudo-colored scans of kidney blood flow in live anesthetized mice. Subsequently, Laser Doppler flowmetry probes were used after laparotomy to obtain kidney blood perfusion values in anesthetized mice. Kidney blood flow measurements and images were acquired using Laser Doppler flowmetry probes (Periflux System 5000) and the Perimed Periscan PIM II Laser Doppler perfusion scanner, respectively. See Supplementary Data for details of how background artifacts were minimized during Laser Doppler flowmetry blood perfusion measurement.

\section{Embryonic Kidney Explant Study}

To examine the effect of cyto-/chemokines on kidney development, embryonic kidneys were obtained from control embryos at E13, as previously described above. After removal from embryos, the embryonic kidneys were cultured following a protocol previously described (14) with slight modifications, as described in Supplementary Data . Either IL-1 $\beta(20 \mathrm{pg} / \mathrm{ml})$, IL-6 (13 pg/ml), IL-12(p70) $(6 \mathrm{pg} / \mathrm{ml})$, or GM-CSF (6 pg/ml) (BioLegend, San Diego, CA) was added to the culture medium of embryonic kidneys. Kidneys were maintained in the culture with cyto-/chemokines for up to $120 \mathrm{~h}$ with the medium changed every $24 \mathrm{~h}$. At the conclusion of culturing, kidneys were stained for SIX2 and calbindin utilizing the wholemount immunohistochemistry protocol described elsewhere (14) and the SIX2 and calbindin antibodies previously described above. After staining, kidney explants were visualized by immunofluorescence microscopy, imaged, and analyzed. For SIX2 analysis, total SIX2+ cells were counted and evaluated for degree of aggregation around calbindin-positive-stained ureteric bud tips. SIX2+ cell counts and degree of aggregation were rated using a 1-10 scale system. A higher score represented greater levels of SIX $2+$ cells and enhanced SIX2+ cell aggregation, whereas a lower score represented decreased SIX2+ cell values and aggregation.

\section{Statistical Analysis}

Sample sizes for all data sets were 5-12 pups, with each pup obtained from a different mother, except data in Figure 1c,d, which had samples sizes of $n \geqslant 23$ and $n \geqslant 57$, respectively. Normality of data in each figure was analyzed using the Shapiro-Wilk test. All data sets 


\section{Articles | Barnett et al.}

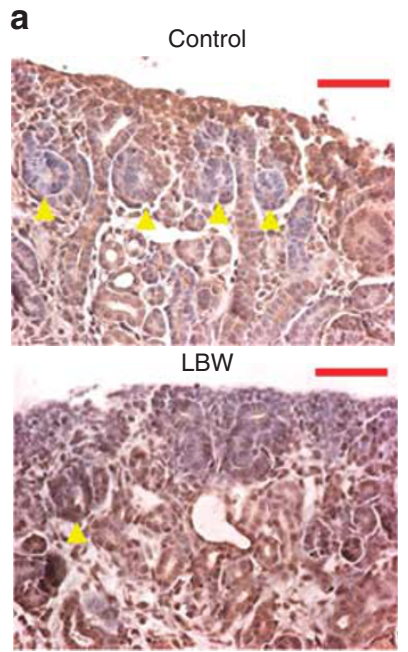

b
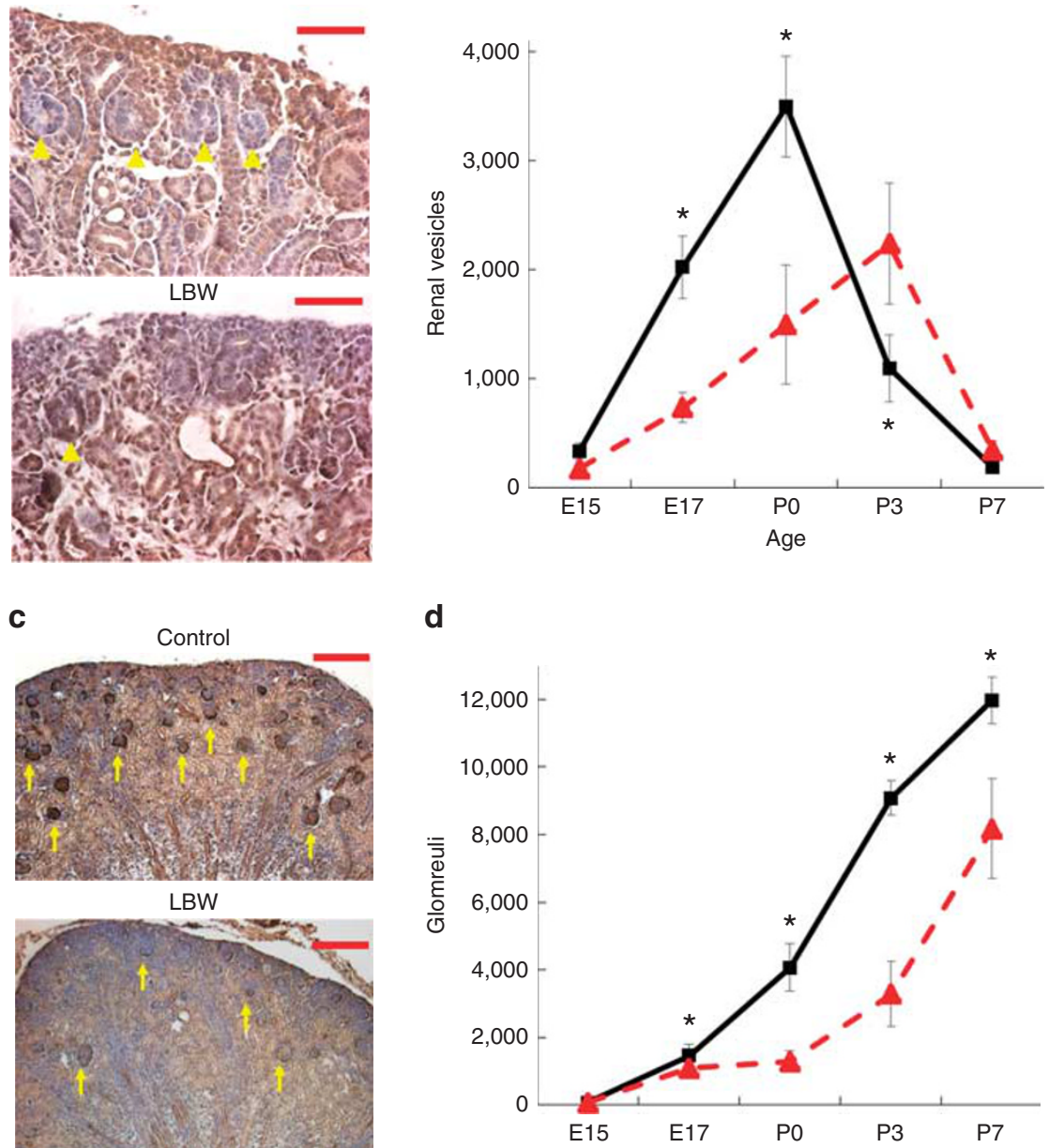

d

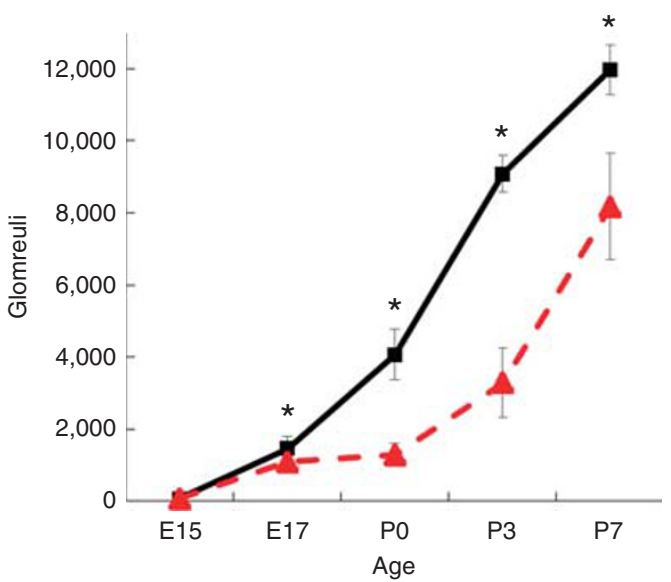

Figure 3. Renal vesicle and glomerulus quantification in the LBW neonate during nephrogenesis. (a) PNA staining of control and LBW embryonic (E17) kidneys demonstrate renal vesicle formation in the outer renal cortex. Magnification $=\times 400$. Bar $=50 \mu \mathrm{m}$. Yellow arrowheads indicate renal vesicles. (b) Quantification of renal vesicles in control (black solid line) and LBW (red cross-hatched line) neonatal kidneys at different ages. (c) PNA staining of control and LBW embryonic (E17) kidneys demonstrate glomeruli in the outer renal cortex. Magnification $=\times 100$. Bar $=100 \mu \mathrm{m}$. Yellow arrows indicate glomeruli. (d) Quantification of glomeruli in control (black solid line) and LBW (red cross-hatched line) neonatal kidneys at different ages. Slight corrections in brightness and contrast were made to images in (a) and (c).

had a normal distribution, except data in Figures 2c and 5. Data presented as a means of a limited number of replicates $(n \leqslant 12)$ were analyzed using nonparametric methods. For comparisons between two groups (Figures 2,3,4,5,6), a Mann-Whitney $U$-test was utilized. Because of the high number of replicates, data in Figure $\mathbf{1 b}$ were analyzed by a parametric paired T-test. A log-rank test was used to evaluate the data in the Kaplan-Meier survival curve (Figure 1c). In Figures 1,2,3,4,5 and $6 \mathrm{a}$, control values were compared with LBW values at each specific age group using pairwise statistical analyses. Similarly, in Figure 6c, control values were compared individually to each treatment group by pairwise statistical analyses. In graphs in Figures $1,2,3$, the second quartile is represented by the median line, with the first and third quartiles represented by box plots. Whiskers represent minimum and maximum data spread in Figures $\mathbf{1}$ and 2; and 5-95\% confidence intervals in Figure 5. Error bars in Figures 3,4,and 6 represent \pm SEM. Statistical analysis softwares used were GraphPad Prism version 6.00 (GraphPad Software, San Diego, CA) and NCSS9 (Kaysville, UT). Differences between control and LBW were considered significant at $P \leqslant 0.05$ (indicated by ${ }^{*}$ ), unless otherwise denoted.

\section{RESULTS}

Preliminary analysis indicated similar kidney developmental impairment between male and female pups when their mothers were placed on the described severe malnourishment diet (as indicated by no statistical difference in total nephron formation or GFR between genders). Consequently, males and females were grouped together in subsequent analyses. Implementation of the described maternal malnourishment diet during the second half of gestation resulted in offspring that were born with $40 \%$ reduced birth weights (Figure 1a,c). One week after birth, the LBW neonates still had reduced body weights by $30 \%$, as compared with control pups (Figure $\mathbf{1 b}$ and $\mathbf{c}$ ). The LBW neonates had a markedly lower percentage of survival, with only $46 \%$ surviving the first week after birth (Figure 1d). The LBW neonates also had decreased kidney blood perfusion of up to $37 \%$ during the first week after birth (Figure 2a,b). Within the 
a
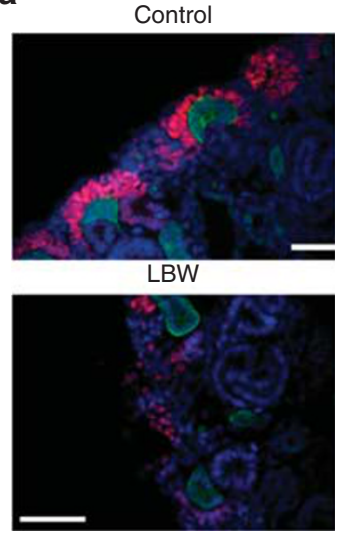

six2/calbindin/DAPI

C

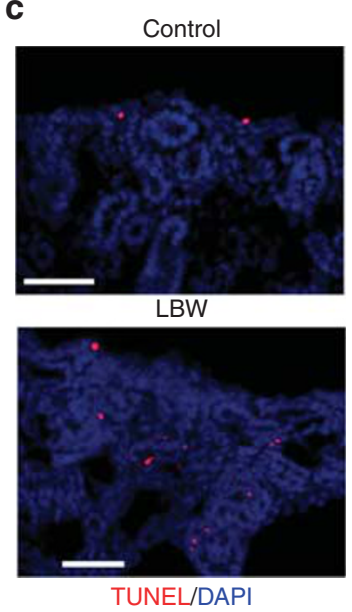

b

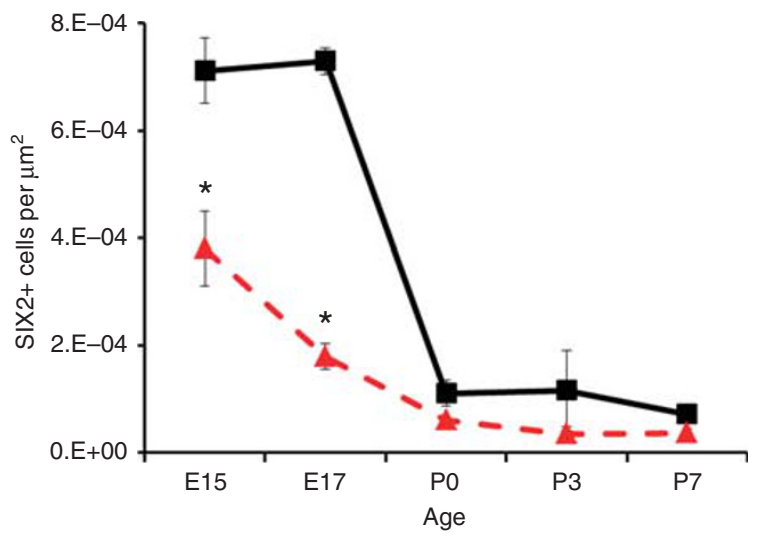

d

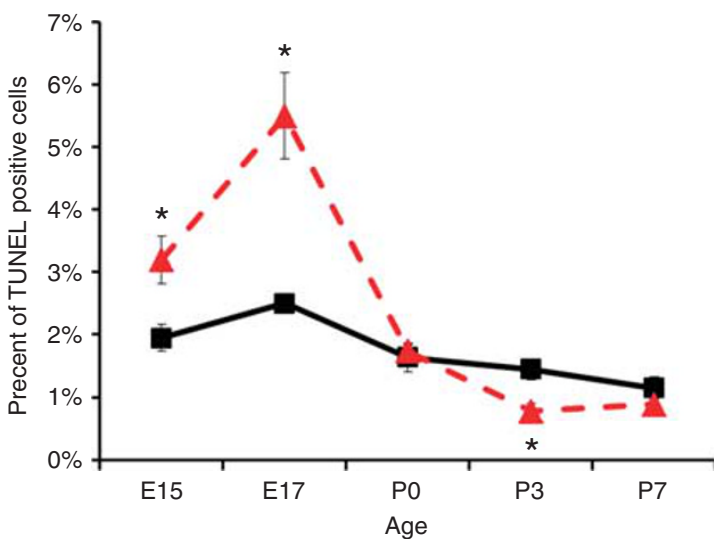

Figure 4. SIX2 and TUNEL positive cell quantification in the outer cortex of the LBW neonatal kidney during nephrogenesis. (a) Immunofluorescence staining with PE (red)-conjugated anti-SIX2 antibodies and FITC (green)-conjugated anti-calbindin antibodies in control and LBW embryonic (E17) kidneys. (b) Quantification of immunofluorescently tagged SIX2+ cells in control (black solid line) and LBW (red cross-hatched line) neonatal kidneys at different ages. (c) Fluorescence TUNEL staining (red) of control and LBW embryonic (E17) kidneys demonstrates apoptosis in the outer renal cortex. For images in (a) and (c): magnification $=\times 400$, Bars $=50 \mu \mathrm{m}$ and nuclei stained blue with DAPI. (d) Quantification of immunofluorescent TUNEL-positive cells in control (black solid line) and LBW (red cross-hatched line) neonatal kidneys at different developmental ages.

first week after birth, LBW neonates displayed reduced kidney function. Within $24 \mathrm{~h}$ of birth, the LBW neonate had a GFR that was 59\% lower than that observed in control pups (Figure 2c). After a 1-week period, GFR had improved, but was still $22 \%$ lower than that in controls. Similarly, total glomerular size (volume) was 69\% lower in LBW neonates, than in controls, on the day of birth (Figure 2d). Despite a rapid increase in size that coincided with the increase in GFR, glomerular volume was still $23 \%$ lower in LBW neonates, than in controls, 1 week later. Results from our mice studies are similar to the reports on LBW human neonates, whose glomerular volume remains reduced even 6 months after birth $(9,15)$. As LBW neonates matured into adults 1 month after birth, both genders experienced elevated mean arterial pressure and fasting blood glucose levels, reduced nephron compliment and GFR, and the kidneys began to show minor mesangial matrix expansion and perivascular fibrosis (Supplementary Figure S1).
In further analysis, we examined kidney development in the maternal malnourished-induced LBW embryo/neonate. We observed that at embryonic day 17 (E17), there was a marked attenuation in the formation of nephron progenitor structures, particularly a significant $64 \%$ decrease in renal vesicle formation (Figure 3a,b) that subsequently led to a $32 \%$ reduction in glomeruli/nephrons at the end of nephrogenesis on P7 (Figure 3c,d). Our results are in accordance with animal studies from other laboratories who have reported that LBW murine neonates resulting from maternal malnourishment have nephron deficits that range from 13 to $41 \%$ (refs 16-18). In addition, though we did not investigate changes in mean arterial pressure in the study here, previous reports indicate LBW rodent models experience an approximate $10-12 \mathrm{~mm} \mathrm{Hg}$ increase in blood pressure as the offspring matures into adulthood (10,16-21). Concurrent with the reduction in renal vesicles at E17, the LBW offspring kidney had a significant decrease of $76 \%$ in SIX2+ 


\section{Articles $\mid$ Barnett et al.}

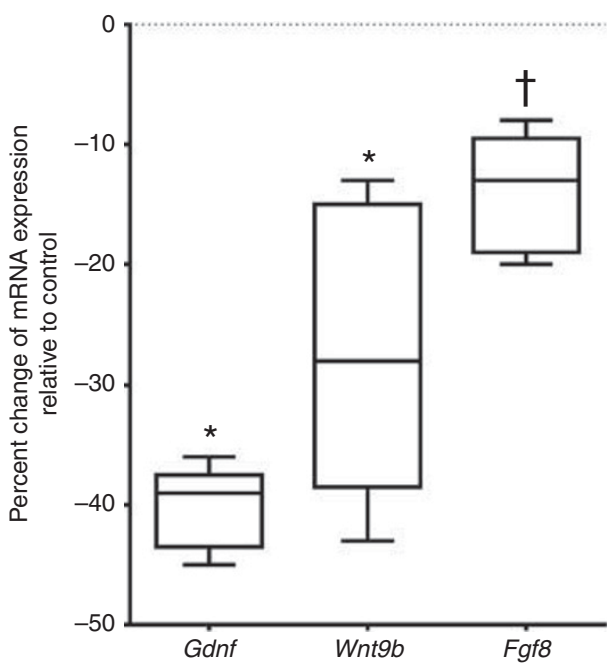

Figure 5. Real-time PCR measurement of Gdnf, Wnt9b, and Fgf8 mRNA expression (normalized to $18 \mathrm{~s}$ rRNA expression) in LBW embryonic (E17) kidneys. Values in the graph are expressed as percentage differences relative to expression in control embryonic (E17) kidneys. ${ }^{\dagger} P=0.11, \mathrm{LBW}$ vs. control.

nephron progenitor cells in the metanephric mesenchyme (aggregated around the tips of the ureteric bud) of the outer cortex (Figure $4 \mathbf{a}, \mathbf{b}$ ). The SIX2+ progenitor cell population is responsible for forming the nephron during renal development. Also at E17, the LBW offspring kidney showed a 2.2 -fold enhancement in apoptosis (Figure 4c,d), suggesting an association between enhanced apoptosis and reduced SIX2+ nephron progenitor cell population.

We conducted a PCR screening of mRNA expression of various critical developmental genes to determine which genes in the developing LBW neonatal kidney are affected during maternal malnourishment. We did not observe any significant modulation in gene expression until age E17. At E17, we found that the expression of the mesenchymal-to-epithelial differentiation signaling factors, Wnt $9 b$ and $F g f$, was decreased by $26 \%$ and $13 \%$, respectively, in the LBW offspring kidney (Figure 5). We also observed that the expression of Gdnf was downregulated in the developing kidney at E17. Other genes that we examined, but that did not demonstrate a statistically significant downregulation in the developing LBW kidney, included Wt1, Wnt4, Sall1, and Bmp7.

On the day of birth, serum samples obtained from LBW neonates demonstrated enhanced levels of pro-inflammatory cyto-/chemokines, including IL-1 $\beta$, IL-6, IL-12(p70), and granulocyte-macrophage colony-stimulating factor (GM-CSF) (Figure 6a). We also observed a trend in the upregulation of these same cyto-/chemokines in the maternal circulation of the malnourished pregnant mother during the end of gestation (data not shown). We questioned whether the increase in these pro-inflammatory cyto-/chemokines could impair nephrogenesis before and/or after birth by adversely affecting SIX2+ cell populations. We used embryonic explanted kidneys taken from embryos at E13 to answer this question. We cultured the developing explanted kidneys with

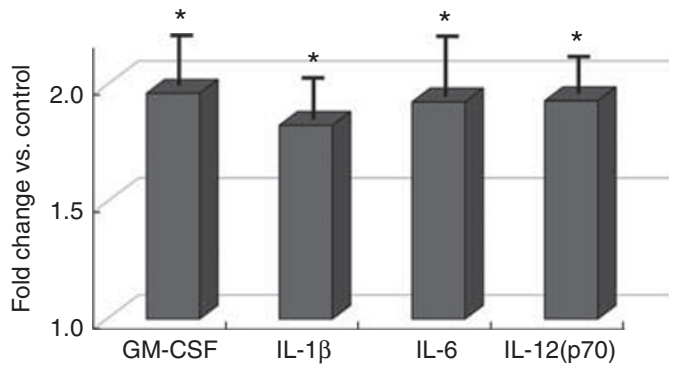

b
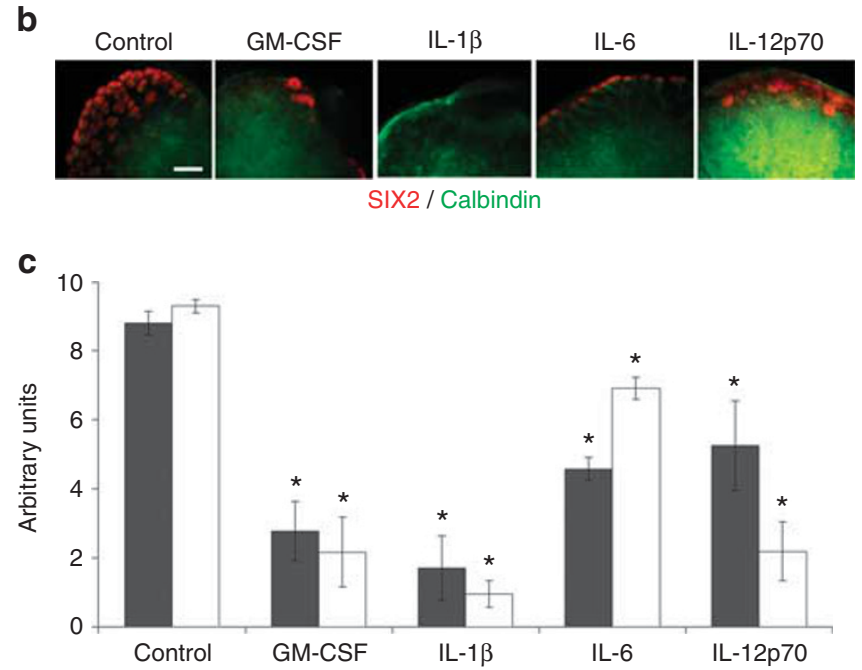

Figure 6. Circulating cyto-/chemokines in the LBW neonate and their effect on renal SIX2+ cells. (a) Luminex measurement of circulating GMCSF, IL-1 3, IL-6, and IL-12(p70) in the LBW neonate on the day of birth. Data are presented as fold change relative to controls. (b) Embryonic explanted kidneys were stained with PE (red)-conjugated anti-SIX2 antibodies and FITC (green)-conjugated anti-calbindin antibodies after culturing with the described cyto-/chemokines for up to $120 \mathrm{~h}$.

Magnification $=\times 100$. Bar $=200 \mu \mathrm{m}$. (c) After culturing with cyto-/ chemokines, embryonic kidney explants were evaluated for SIX2+cell quantity (solid gray bars) and aggregation (solid white bars).

the observed pathological concentrations of IL-1 $\beta$, IL-6, IL-12 (p70), and GM-CSF for up to $120 \mathrm{~h}$. The cyto-/chemokines severely reduced levels of SIX $2+$ nephron progenitor cells and decreased aggregation of these cells in the kidney explants (Figure $\mathbf{6 b}, \mathbf{c}$ ). The greatest reduction of SIX $2+$ cells in the kidneys was observed upon administration of IL-1 $\beta$ and GM-CSF. Thus, at the time of birth, enhanced circulating pro-inflammatory factors further impair nephrogenic processes in the LBW kidney and continue to blunt nephron and glomeruli formation.

\section{DISCUSSION}

Maternal (22) and paternal (23) malnutrition prior to conception is associated with increased risk of disease in the resulting offspring. After conception, proper maternal nutrition during pregnancy is very critical for normal embryonic renal development. In addition to their use as building blocks for tissues, amino acids have various critical roles for normal embryonic development. For instance, arginine has been demonstrated to enhance fetal weight gain and birth weight 
by improving uteroplacental perfusion and subsequent fetal nutrient delivery via upregulation of local nitric oxide (24). In addition to protein deficiency, the observed effects in our study may also be due to a deficiency in other nutrients caused by the implemented restricted diet. Adequate supply of vitamins and minerals, such linoleic/linolenic acid, vitamin A, iron, zinc, and folate, have shown to be critical for normal developmental signaling, structural formation and enzymatic activity (25). Furthermore, deficiencies in folate, vitamin B, methionine, and other amino acids and minerals alter DNA methylation and epigenetic patterns during development, resulting in altered gene expression (26). These factors, including amino acids critical for renal development, have previously shown to be reduced in the fetal circulation, allantoic, and amniotic fluids (27) during maternal malnourishment.

GFR results from our murine LBW neonates differ somewhat from previous reports in human LBW neonates. In contrast to our report that GFR is decreased the first week after birth in the mouse LBW neonate, as compared with controls, two recent reports suggest that although there is a trend for GFR reduction in LBW human neonates, this decrease is not statistically different from controls $(9,15)$. Although this difference may be due to species-dependent differences in GFR response to LBW, we suspect that the discrepancy may be because of the severity of our LBW model. In the previous human reports, LBW neonates had a glomerular volume that was reduced by $\sim 25-$ $30 \%$ after birth, whereas mice LBW neonates in our study experienced a much more severe $69 \%$ reduction in glomerular size. To overcome reduced nephron endowment and to maintain a normal overall GFR, enhanced single-nephron GFR compensation typically occurs through hyperfiltration in humans. However, because of the severe decrease in volume and maturation of glomeruli in LBW neonates in our murine model, hyperfiltration compensation may not be able to fully compensate and increase GFR until glomeruli and kidneys are more fully developed. In support of this rationale, we observed, in our murine LBW model, a significant increase in GFR during the first week after birth as glomeruli matured and volume increased.

The extent of impaired embryonic kidney development is not only dependent on the severity of protein or caloric deficiency but also on the timing of protein/caloric restriction during gestation and on offspring gender. Gestational malnourishment during the period of ongoing nephrogenesis has a much greater impact on final nephron complement and mean arterial pressure, as compared with malnourishment, which is limited to periods when nephrogenesis is not ongoing (28-31). In mice embryos, nephrogenesis occurs during the second half of gestation, with over $90 \%$ of nephrons formed after E15 (19). We observed a similar exponential increase in renal vesicle and glomeruli formation late in gestation beginning at E17 in control mice, an effect that was markedly blunted when pregnant mothers were malnourished during this gestational period. Furthermore, fetal programming associated with maternal malnourishment occurs in offspring in a gender-dependent manner (32). Female offspring are resistant to the nephrogenesis impairment and rise in blood pressure that occur in male offspring during mild-to-moderate gestational malnourishment. However, more severe maternal malnourishment (as used in our study here) reduces nephron numbers and raises blood pressure similarly in both LBW female and male neonates (Supplementary Figure S1) $(21,29,30)$.

During maternal malnourishment, we observed that multiple events occur at day E17 in the developing embryo, which affects nephrogenesis. At E17, during maternal malnourishment there is a reduction of metanephric mesenchymal SIX2+ cells, which was accompanied by a concomitant increase in apoptosis and severe attenuation of mesenchymal aggregation into renal vesicles in the developing kidney. Two recent developmental studies that used a maternal malnourishment model reported a potential link between enhanced apoptosis in the embryonic kidney and reduced progenitor pool size. In these two studies, malnourishment of pregnant mice was associated with a twofold increase in apoptosis in the embryonic kidney (21), effectively reducing metanephric cells by $50 \%$ (ref. 21 ) and blunting ureteric bud branching by $28 \%$ (ref. 33). Genetic experiments by Cebrian et al. (8) further illustrated that a reduction in nephron progenitor cells results in reduced nephron endowment. In experiments by Cebrian et al. (8), genetic ablation of fetal nephron progenitor cells by $40 \%$ severely limited ureteric branching and reduced adult nephron endowment by $42 \%$.

Expression of Wnt9b, Fgf8, and Gdnf was downregulated at E17. During nephrogenesis, Wnt9b expression is normally enhanced in the developing kidney and signals SIX2+ stem cells of the metanephric mesenchyme to undergo two crucial processes-proliferative self-renewal and differentiation into early nephron structures including renal vesicles (34). Reduced $W n t 9 b$ expression would expectedly impede nephrogenesis by blunting formation of early nephron progenitor structures. In addition, reduced Fgf8 expression, as occurs during malnourishment, has shown to prevent progression of nephrogenesis, promote nephron progenitor cell death, and arrest kidney development $(35,36)$. Fgf8 is expressed in early nephron structures and is essential for transition of pretubular aggregates and renal vesicles to more mature nephron structures, such comma- and S-shaped bodies. Furthermore, Fgf 8 expression is also essential for cell survival at distinct steps of nephrogenesis (35). GDNF is released by stem cells of the metanephric mesenchyme during development and signals the ureteric bud to branch and invade the metanephric mesenchyme. The reduced expression of Gdnf suggests that during malnourishment, GDNF signaling is compromised in the impaired SIX2+ population of the developing kidney.

In addition to the direct effects associated with nutrient and protein deficiency, stress-induced factors upregulated in the maternal and/or embryonic/neonate circulation can also blunt nephrogenesis. Metabolic stress, such as that associated with malnourishment, alters various hormonal and inflammatory molecules in the LBW neonate and in the maternal circulation 
(37). Because of malnourishment-associated inadequacies in the placental barrier, many of these molecules generated in the pregnant mother, including upregulated pro-inflammatory cyto-/chemokines, are able to cross the placenta and enter the fetal circulation (38). Consequently, inflammatory cyto-/chemokines from both the mother and the LBW neonate can impede nephrogenesis in the developing kidney by reducing SIX $2+$ progenitor cell quantity and aggregation.

\section{SUPPLEMENTARY MATERIAL}

Supplementary material is linked to the online version of the paper at http://www.nature.com/pr

\section{STATEMENT OF FINANCIAL SUPPORT}

Studies were supported by AHA grant 12SDG9080006, ASN grant 010973101, and The New York Community Trust-Renal Clinical Fund (BBR).

Disclosure: The authors declare no conflict of interest.

\section{REFERENCES}

1. Hamilton BE, Martin JA, Osterman MJ, Curtin SC, Matthews TJ. Births final data for 2014. Natl Vital Stat Rep 2015;64:1-64.

2. Gillman MW. Developmental origins of health and disease. N Engl J Med 2005;353:1848-50.

3. Godfrey KM, Barker DJ. Fetal nutrition and adult disease. Am J Clin Nutr 2000;71:1344S-52S.

4. Vikse BE, Irgens LM, Leivestad T, Hallan S, Iversen BM. Low birth weight increases risk for end-stage renal disease. J Am Soc Nephrol 2008;19: 151-7.

5. Khalsa DD, Beydoun HA, Carmody JB. Prevalence of chronic kidney disease risk factors among low birth weight adolescents. Pediatr Nephrol 2016;31:1509-6.

6. Luyckx VA, Brenner BM. Birth weight, malnutrition and kidney-associated outcomes-a global concern. Nat Rev Nephrol 2015;11:135-49.

7. Hodgin JB, Rasoulpour M, Markowitz GS, D'Agati VD. Very low birth weight is a risk factor for secondary focal segmental glomerulosclerosis. Clin J Am Soc Nephrol 2009;4:71-6.

8. Cebrian C, Asai N, D'Agati V, Costantini F. The number of fetal nephron progenitor cells limits ureteric branching and adult nephron endowment. Cell Rep 2014;7:127-37.

9. Iyengar A, Nesargi S, George A, Sinha N, Selvam S, Luyckx VA. Are low birth weight neonates at risk for suboptimal renal growth and function during infancy? BMC Nephrol 2016;17:100.

10. Cullen-McEwen LA, Douglas-Denton RN, Bertram JF. Estimating total nephron number in the adult kidney using the physical disector/ fractionator combination. Methods Mol Biol 2012;886:333-50.

11. Cullen-McEwen LA, Armitage JA, Nyengaard JR, Moritz KM, Bertram JF. A design-based method for estimating glomerular number in the developing kidney. Am J Physiol Renal Physiol 2011;300:F1448-53.

12. Schreiber A, Shulhevich Y, Geraci S, et al. Transcutaneous measurement of renal function in conscious mice. Am J Physiol Renal Physiol 2012;303: F783-8.

13. Schock-Kusch D, Sadick M, Henninger N, et al. Transcutaneous measurement of glomerular filtration rate using FITC-sinistrin in rats. Nephrol Dial Transplant 2009;24:2997-3001.

14. Barak H, Boyle SC. Organ culture and immunostaining of mouse embryonic kidneys. Cold Spring Harb Protoc 2011;2011: pdb prot5558.

15. Kandasamy Y, Smith R, Wright IM, Lumbers ER. Relationships between glomerular filtration rate and kidney volume in low-birth-weight neonates. J Nephrol 2013;26:894-8.
16. Brennan KA, Kaufman S, Reynolds SW, et al. Differential effects of maternal nutrient restriction through pregnancy on kidney development and later blood pressure control in the resulting offspring. Am J Physiol Regul Integr Comp Physiol 2008;295:R197-205.

17. Langley-Evans SC, Langley-Evans AJ, Marchand MC. Nutritional programming of blood pressure and renal morphology. Arch Physiol Biochem 2003;111:8-16.

18. Langley-Evans SC, Welham SJ, Jackson AA. Fetal exposure to a maternal low protein diet impairs nephrogenesis and promotes hypertension in the rat. Life Sci 1999;64:965-74.

19. Short KM, Combes AN, Lefevre J, et al. Global quantification of tissue dynamics in the developing mouse kidney. Dev Cell 2014;29:188-202.

20. Woods LL. Maternal nutrition and predisposition to later kidney disease. Curr Drug Targets 2007;8:906-13.

21. Welham SJ, Wade A, Woolf AS. Protein restriction in pregnancy is associated with increased apoptosis of mesenchymal cells at the start of rat metanephrogenesis. Kidney Int 2002;61:1231-42.

22. Barker DJ. The developmental origins of adult disease. J Am Coll Nutr 2004;23:588S-95.

23. Li J, Tsuprykov O, Yang X, Hocher B. Paternal programming of offspring cardiometabolic diseases in later life. J Hypertens 2016;34:2111-6.

24. Xiao XM, Li LP. L-Arginine treatment for asymmetric fetal growth restriction. Int J Gynaecol Obstet 2005;88:15-8.

25. McArdle HJ, Ashworth CJ. Micronutrients in fetal growth and development. Br Med Bull 1999;55:499-510.

26. Park LK, Friso S, Choi SW. Nutritional influences on epigenetics and agerelated disease. Proc Nutr Soc 2012;71:75-83.

27. Wu G, Pond WG, Ott T, Bazer FW. Maternal dietary protein deficiency decreases amino acid concentrations in fetal plasma and allantoic fluid of pigs. J Nutr 1998;128:894-902.

28. Woods LL, Weeks DA. Prenatal programming of adult blood pressure: role of maternal corticosteroids. Am J Physiol Regul Integr Comp Physiol 2005;289:R955-62.

29. Woods LL, Weeks DA, Rasch R. Programming of adult blood pressure by maternal protein restriction: role of nephrogenesis. Kidney Int 2004;65: 1339-48.

30. Vehaskari VM, Aviles DH, Manning J. Prenatal programming of adult hypertension in the rat. Kidney Int 2001;59:238-45.

31. Langley-Evans SC, Welham SJ, Sherman RC, Jackson AA. Weanling rats exposed to maternal low-protein diets during discrete periods of gestation exhibit differing severity of hypertension. Clin Sci 1996;91:607-15.

32. Reichetzeder C, Dwi Putra SE, Li J, Hocher B. Developmental origins of disease - crisis precipitates change. Cell Physiol Biochem 2016;39:919-38.

33. Mesquita FF, Gontijo JA, Boer PA. Maternal undernutrition and the offspring kidney: from fetal to adult life. Braz J Med Biol Res 2010;43: 1010-8.

34. Karner CM, Das A, Ma Z, et al. Canonical Wnt9b signaling balances progenitor cell expansion and differentiation during kidney development. Development 2011;138:1247-57.

35. Grieshammer U, Cebrian C, Ilagan R, Meyers E, Herzlinger D, Martin GR. FGF8 is required for cell survival at distinct stages of nephrogenesis and for regulation of gene expression in nascent nephrons. Development 2005;132:3847-57.

36. Perantoni AO, Timofeeva O, Naillat F, et al. Inactivation of FGF8 in early mesoderm reveals an essential role in kidney development. Development 2005;132:3859-71.

37. Kanitz E, Otten W, Tuchscherer M, et al. High and low proteinratio carbohydrate dietary ratios during gestation alter maternal-fetal cortisol regulation in pigs. PLoS ONE 2012;7:e52748.

38. Thornburg KL, O'Tierney PF, Louey S. Review: the placenta is a programming agent for cardiovascular disease. Placenta 2010;31 (Suppl): S54-9. 